\title{
Determination of Isoniazid Acetylator Phenotype and its Clinical Implication in Rwandan Tuberculosis Patients
}

\author{
Mukanyangezi M. F ${ }^{1}$, Kadima N. ${ }^{1^{*}}$, Musemakweri $\mathrm{A}^{2}$ \\ ${ }^{1}$ Department of Pharmacology, School of Medicine and Pharmacy, University of Rwanda, P.O. Box 117, Huye Rwanda \\ ${ }^{2}$ University Teaching Hospital of Butare, Department of Internal Medicine, P.O. Box 117, Huye Rwanda
}

\begin{abstract}
The purpose was to determine the normal profile of the isoniazid acetylator phenotype, the effect of tuberculosis (TB) on the profile, and the impact of the profile on early clinical responses following a treatment with the first line drug regimen. The study sample comprised 20 healthy volunteers and 22 TB naive patients. The tests for TB and HIV were conducted in the laboratory of the University Teaching Hospital Butare (CHUB). The phenotype was achieved by measuring the percentage of acetylisoniazid in the urine after a unique oral dose of isoniazid $10 \mathrm{mg} / \mathrm{kg}$. TB patients were treated for two months with standard regimen combining rifampicin, isoniazid, pyrazinamide and ethambutol. A trimodal distribution profile was observed in both the healthy and tuberculosis groups, giving $30 \%$ versus $13.6 \%$ as slow acetylators, $45 \%$ versus $54.5 \%$ as intermediate acetylators, and $25 \%$ versus $23.2 \%$ as fast acetylators respectively. After a 2 -month treatment of the TB patients, the sputum smear cultures were negative in about $81 \%$ independent of the acetylator or HIV status. Early side effects experienced were dominated by peripheral neuropathies mostly in slow and intermediate acetylators.
\end{abstract}

Key words: Acetylator, isoniazid, phenotype, tuberculosis, HIV, Rwanda

\section{Introduction}

Tuberculosis (TB) is an old infectious and contagious disease caused by the micro-organism Mycobacterium tuberculosis (MTB) that most often affects the lungs. Over nine million cases of active TB occur in the world each year causing about three million deaths which represent about $25 \%$ of all "avoidable deaths", of which over $95 \%$ occur in low- and middle-income countries (GHO, 2013). The recent invasion of human immunodeficiency virus (HIV) has worsened the recurrence of TB worldwide as it has been observed that people with HIV are estimated to have a 20-37 times greater likelihood of developing active TB disease once infected with MTB (WHO,2013a). The worst aspect of it is that multi-drug-resistant TB (MDRTB) is a challenge in virtually all countries already affected (WHO, 2013b; Pasipanodya et al., 2012, pp.169-177). According to the Rwanda Ministry of Health $(\mathrm{MoH}$, 2012) annual report, at least 7,175 TB cases were recorded in 2011 and the seroprevalence of HIV among TB patients was $28 \%$.

Multiple strategies have been defined and implemented with the objective to stop TB and HIV related deaths (GPS-TB, 2013), and among them the greatest emphasis has been put on patient management by using efficient medications. It appeared obvious that while TB is a serious scourge, deaths are reduced if treatments are completed with a long 6 months course of a combination of four to six antibiotics (WHO, 2010). Many factors however, can influence the completeness of treatment of which patient's adherence, side effects and adequacy of drug dosing play a key role (Kaishusha \& Kadima, 2009, pp.205-215; NIH, 2013). Numerous studies have been reported in the literature demonstrating how an individual's genetic profile may be used for adjusting drug dosages and choosing a medication with minimal side effects to promote the patient's compliance (Kinzig-Schippers et al., 2005, 1733-1738). The polymorphism profile of isoniazid acetylation varies with race and ethnicity and its knowledge has been found useful in tailoring individual dosing to minimize side effects and maximize clinical outcomes (Junichi et al., 2013, pp.1091-1101; Patin et al., 2006, p.720; Ellard, G.A., 1976, pp.610-625).

In Rwanda no study has been done to handle data on the polymorphism in drug metabolism. The search of information on how the general population of Rwanda genetically metabolise drugs was thus a matter of concern to ensure the safety and effectiveness of drugs used. To date no study has been undertaken in the Rwandan population regarding this issue, we designed this pilot study for a small number of healthy individuals and tuberculosis patients to determine the normal metabolism profile, the effect of TB on the normal rate, and the impact of

*Corresponding author: kntokamunda@ur.ac.rw 
the profile on the early clinical responses to the first line therapeutic regimen.

\section{Methods}

\section{Study site, subjects, and sample}

The study was conducted at the University Teaching Hospital of Butare (UTHB), which is one of the referral hospitals in Rwanda, located in the Southern Province, with a capacity of 500 beds. The UTHB has 20 clinical services including an infections unit for TB and HIV voluntary counselling, testing and treatment. Over a period of one month and on the day of admission, 22 treatment naive TB patients of both sexes attending the UTHB and 20 healthy volunteers (medically examined and confirmed HIV negative) also of both sexes were recruited from among students of the former National University of Rwanda (NUR), currently University of Rwanda Huye Campus. All patients had to be TB positive proven by microscopy and smear culture, and HIV positive proven by the ELISA method (Van de Perre et al.,1991, pp.593598) and Western blot, had to be naive, meaning have never received TB or HIV medication before.

\section{Study design}

Age, sex, weight, nutrition, and HIV status were recorded on the day of entering the study using a data collection form. Nutritional status was estimated by both the clinical assessment (hair, mouth, eyes, skin) and the anthropometric method relating weight-height as compared to normal body mass index (BMI: 18.5-24.5). Those with bad clinical signs or a BMI less than $70 \%$ of normal were qualified as "bad status". Each participant, on an empty stomach in the morning and free of any medicine for at least 48 hours was given $10 \mathrm{mg}$ isoniazid (INH) per $\mathrm{kg}$ of body weight. Six to 8 hours after dosing, participants were asked to collect complete urine samples. The urine samples collected were routed quickly to the medical laboratory, and kept cool until analysis. Healthy volunteers were received in group and immediately released thereafter. TB patients were naïve patients. Each patient was received and treated individually on the first day of consultation. After the collection of urine specimens, each patient was admitted at CHUB and treated using standard regimen combining four drugs: RHZ (rifampicin $120 \mathrm{mg}$ /isoniazid $50 \mathrm{mg} /$ pyrazinamide $300 \mathrm{mg}$ ); Ethambutol $400 \mathrm{mg}$. They were each hospitalized for a two months Directly Observed Short Course Therapy (DOTS). During the first two months, they were measured for clinical improvement by physical examination and microbial clearance by routine sputum culture at the end of treatment. There were also monitored for side effects.

\section{Phenotyping method}

The phenotying determination was carried out by a simple spectrophotometric method (Eidus \& Hodgkin, 1973, pp.130-133; Weber et al. 1974, pp.467-473. After a test dose of isoniazid, free isoniazid (INH) and its acetyl derivative $(\mathrm{AcINH})$ were estimated in urine by the same colorimetric reaction. The classification into slow acetylator (SA) and fast acetylator (FA) was based on the ratio of the metabolite acetylisoniazid to the total hydrazines excreted ( $\%$ AcINH). The method as described in the original paper is as following:

"Laboratory procedure: To two 1-ml aliquots of urine, $0.5 \mathrm{ml}$ of $0.5 \mathrm{~N}$ hydrochloric acid is added and kept at room temperature for $15 \mathrm{~min}$. One drop of reagent grade acetic anhydride is delivered to one of the aliquots and shaken for one minute, followed by one drop of $7 \mathrm{~N}$ sodium hydroxide. Two drops of distilled water are added to the other aliquot to ensure equal volumes. Both aliquots are then neutralized by $0.5 \mathrm{ml}$ of $0.5 \mathrm{~N}$ sodium hydroxide.

Estimation of the acetylisoniazid concentration: To the above-mentioned aliquots reagents are added successively, as follows: (a) $1 \mathrm{ml}$ of $0.5 \mathrm{M}$ potassium phosphate buffer, pH 6 (prepared by mixing $87.7 \mathrm{ml}$ of $0.5 \mathrm{M}$ potassium dihydrophosphate and $12.3 \mathrm{ml}$ of $0.5 \mathrm{M}$ potassium hydrophosphate); (b) $1 \mathrm{ml}$ of a 20\% aqueous solution of potassium cyanide (prepared daily); (c) $4 \mathrm{ml}$ of a 12.5 $\%$ solution of chloramine $\mathrm{T}$ (prepared daily), with shaking; and (d) after a waiting period of $1 \frac{1}{2} / 2 \mathrm{~min}, 5 \mathrm{ml}$ of acetone, reagent grade, with thorough mixing. Slight precipitation may be occasionally noticed in concentrated urine samples and can be cleared by centrifugation. Optical density readings of the aliquots are undertaken at wavelength $550 \mathrm{~nm}$. Classification of slow and fast inactivators: Two different scales can be used for group identification of inactivators-namely, (1) the inactivation index (proportions of acetylisoniazid and free isoniazid in $\mathrm{mg} /$ litre): calculation of acetylisoniazid as a proportion of the total hydrazides excreted in the urine. Patients yielding a proportion lower than $70 \%$ are considered as slow inactivators, whereas rapid acetylators produce values over $75 \%$ with this method."

\section{Data Analysis}

Data were computerized and analyzed with SPSS v.16 statistical package and Microsoft Excel 2007 software for Windows. Where it is required, data were given as mean \pm SEM. Differences between groups or the interrelation- 
ship between parameters were considered significant at $\mathrm{p}$-value $=0.05$ (Pearson Chi-square test) or Fisher's exact test.

\section{Ethical issues}

Each participant voluntarily entered the study upon agreed informed consent. The protocol was cleared by the Ethics Committee of Butare University Teaching Hospital.

\section{Results}

\section{Baseline characteristics of patients}

Characteristics of participants are described in Table 1. For the entire sample, males represented $54.76 \%$ and females $45.24 \%$. The mean age was $28.35 \pm 3.42$ [23-38] years in healthy volunteers and $31.36 \pm 9.96$ [16-51] in TB-patients, while the mean body weight was $64.15 \pm$ 9.05 [55-80] kg and $51.55 \pm 9.43$ [40-63] kg respectively. Among TB patients, $8(36.36 \%)$ were in an undernourished state $(\mathrm{BMI}<18)$, while $6(27.27 \%)$ were HIV-infected.

\section{Acetylator phenotype profile}

The distribution profile of $\%$ AcINH is presented in Fig.1. It can be split into three clusters corresponding to a trimodal profile. The three classes were defined as Slow-Acetylator (SA), Intermediate-Acetylator (IA), and Fast-Acetylator (FA) according to whether the $\%$ AcINH fell into one of the three clusters: $25-45 \%, 46-75 \%$, or $76-98 \%$. The IA group is the most represented, overlapping SA and FA groups in both healthy volunteers and
TB patients. The values of $\%$ AcINH in the middle cluster were shifted to the right in TB patients compared to healthy persons. However, according to the method used, taking $70 \%$ of AcINH as the cut-point, the bimodal profile would be considered. The frequencies for either trimodal or bimodal distribution are presented in Table 2 .

\section{Factors influencing acetylation profile}

The influence of demographic characteristics on the acetylation phenotype as tested in the group of TB-patients is shown in Table 3 showing that all variables, but the nutritional status $(p=0.015)$ and age $(p=0.031)$, had no significant effect. Here, only the bimodal profile was used for comparison.

\section{Effect of Acetylator profile on Clinical outcomes}

During the two-month treatment with RHZE combination, the efficacy and tolerance of the treatment was monitored by sputum smears cultures, relief of clinical symptoms and side effects experienced (Table 4). Eighteen patients whose sputum smears cultures were previously tested positive showed a negative result at the control test after two months and this independently of their acetylator or HIV status ( $p>0.05)$. The general physical health state was graded as improved in $77.3 \%$ of cases. One patient of the cohort died in pulmonary complications while $3(13.6 \%)$ remained in stable condition without significant improvement and with smear culture positive. The adverse reactions observed were essentially dominated by the peripheral neuropathies and skin reactions.

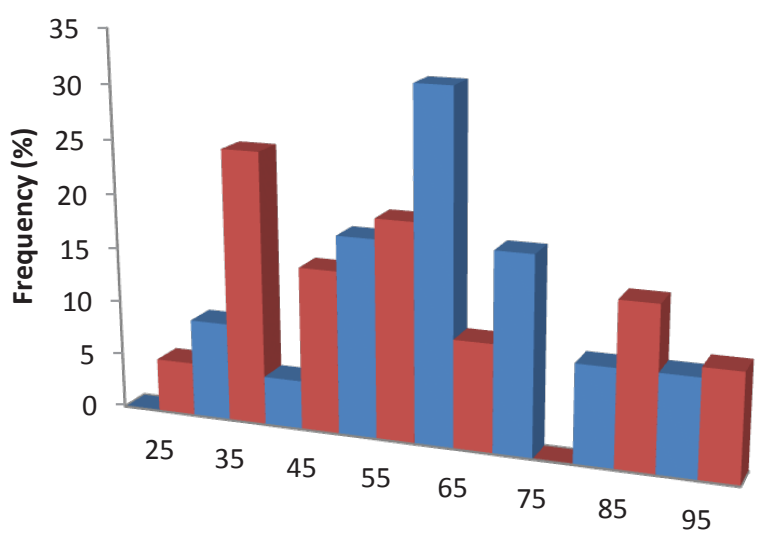

\%Acetylisoniazid in urine

Figure 1. Histogram distribution profile of INH phenotyping in all 40 individuals (20 healthy and 20 TB patients) 
Table 1. Distribution of participants on baseline characteristics

\begin{tabular}{|c|c|c|c|c|c|c|c|}
\hline \multirow[t]{2}{*}{ Variable } & \multirow[t]{2}{*}{ Category } & \multicolumn{2}{|c|}{$\begin{array}{c}\text { Healthy } \\
n=20\end{array}$} & \multicolumn{2}{|c|}{$\begin{array}{l}\text { TB-group } \\
n=22\end{array}$} & \multicolumn{2}{|c|}{ Total $=42$} \\
\hline & & $\mathrm{n}$ & $(\%)$ & $\mathbf{n}$ & $(\%)$ & $\mathrm{n}$ & $\%$ \\
\hline \multirow[t]{2}{*}{ Gender } & Male & 10 & 50.0 & 13 & 59.1 & 23 & 54.8 \\
\hline & Female & 10 & 50.0 & 9 & 40.9 & 19 & 45.2 \\
\hline \multirow[t]{3}{*}{ Age (years) } & $16-20$ & 0 & 0 & 5 & 22.7 & 5 & 11.9 \\
\hline & $21-40$ & 20 & 100.0 & 12 & 54.6 & 32 & 76.2 \\
\hline & $41-51$ & 0 & 0 & 5 & 22.7 & 5 & 11.9 \\
\hline \multirow[t]{4}{*}{ Marital status } & Single & 20 & 100.0 & 11 & 50.0 & 31 & 73.8 \\
\hline & Married & 0 & 0 & 7 & 31.8 & 7 & 16.6 \\
\hline & Divorced & 0 & 0 & 2 & 9.1 & 2 & 4.8 \\
\hline & Widow & 0 & 0 & 2 & 9.1 & 2 & 4.8 \\
\hline \multirow[t]{5}{*}{ Occupation } & Student & 20 & 100.0 & 7 & 31.8 & 27 & 64.3 \\
\hline & Farmer & 0 & 0 & 8 & 36.4 & 8 & 19.0 \\
\hline & Military & 0 & 0 & 4 & 18.2 & 4 & 9.5 \\
\hline & Driver & 0 & 0 & 2 & 9.1 & 2 & 4.8 \\
\hline & Prisoner & 0 & 0 & 1 & 4.5 & 1 & 2.4 \\
\hline \multirow[t]{3}{*}{ Weight (kg) } & $35-49$ & 0 & 0 & 8 & 36.7 & 8 & 19.1 \\
\hline & $50-69$ & 12 & 60.0 & 13 & 59.1 & 25 & 59.5 \\
\hline & $70-85$ & 8 & 40.0 & 1 & 4.6 & 9 & 21.4 \\
\hline \multirow[t]{2}{*}{ Nutrition } & Normal & 20 & 100.0 & 14 & 63.6 & 34 & 81.0 \\
\hline & $\mathrm{Bad}$ & 0 & 0 & 8 & 36.4 & 8 & 19.0 \\
\hline \multirow[t]{2}{*}{ HIV } & $\mathrm{HIV}+$ & 0 & 0 & 6 & 27.3 & 6 & 14.3 \\
\hline & HIV- & 20 & 100.0 & 16 & 72.7 & 36 & 85.7 \\
\hline \multirow[t]{2}{*}{ Site of TB } & Pulmonary & 0 & 0 & 19 & 86.4 & 19 & 42.2 \\
\hline & Extrapulmonary & 0 & 0 & 3 & 13.6 & 3 & 7.1 \\
\hline
\end{tabular}

Table 2. Frequencies of acetylation profile calculated under Trimodal or Bimodal distribution

\begin{tabular}{lllllllll}
\hline Model & Phenotype & \multicolumn{2}{c}{ Healthy } & \multicolumn{2}{c}{ TB } & \multicolumn{3}{c}{ Both } \\
& & $\mathbf{n}$ & $\mathbf{\%}$ & $\mathbf{n}$ & $\mathbf{\%}$ & $\mathbf{n}$ & $\mathbf{\%}$ & p-value* \\
\hline \multirow{2}{*}{ Trimodal } & Slow Acetylator & 6 & 30.0 & 3 & 13.6 & 9 & 21.4 & 0.432 \\
& Intermediate Acetylator & 9 & 45.0 & 12 & 54.5 & 21 & 50.0 & \\
& Fast Acetylator & 5 & 25.0 & 7 & 31.8 & 12 & 28.6 & \\
\multirow{3}{*}{ Bimodal } & Slow Acetylator & 15 & 75.0 & 15 & 68.2 & 29 & 69.0 & 0.625 \\
& Rapid acetylator & 5 & 25.0 & 7 & 31.8 & 13 & 31.0 & \\
\hline
\end{tabular}

${ }^{*}$ Pearsons Chi-square, 95\% confidence limit 
Table 3. Factors that can influence the phenotype profile in $22 \mathrm{~TB}$ patients

\begin{tabular}{|c|c|c|c|c|c|c|}
\hline \multirow[b]{2}{*}{ Indicator } & \multirow[b]{2}{*}{ Category } & \multicolumn{2}{|c|}{$\begin{array}{c}\text { Slow acetylator } \\
(n=15)\end{array}$} & \multicolumn{2}{|c|}{$\begin{array}{c}\text { Fast acetylator } \\
(\mathrm{n}=7)\end{array}$} & \multirow[b]{2}{*}{$p$-value } \\
\hline & & $\mathbf{n}$ & $\%$ & $\mathrm{n}$ & $\%$ & \\
\hline \multirow[t]{2}{*}{ Nutrition status } & Normal & 7 & 46.7 & 7 & 100.0 & 0.015 \\
\hline & $\mathrm{Bad}$ & 8 & 53.3 & 0 & 0.0 & \\
\hline \multirow[t]{2}{*}{ HIV test } & Negative & 10 & 66.7 & 6 & 85.7 & 0.350 \\
\hline & Positive & 5 & 33.3 & 1 & 14.3 & \\
\hline \multirow[t]{3}{*}{ Age index (years) } & $16-20$ & 1 & 6.7 & 4 & 57.1 & 0.031 \\
\hline & $21-49$ & 10 & 66.7 & 2 & 28.6 & \\
\hline & $>50$ & 4 & 26.7 & 1 & 14.3 & \\
\hline \multirow[t]{3}{*}{ Weight index $(\mathrm{kg})$} & $<40$ & 5 & 33.3 & 1 & 14.3 & 0.417 \\
\hline & $40-69$ & 8 & 53.3 & 6 & 85.7 & \\
\hline & $>70$ & 2 & 13.3 & 0 & 0.0 & \\
\hline \multirow[t]{2}{*}{ Sex } & Female & 7 & 46.7 & 2 & 28.6 & 0.606 \\
\hline & Male & 8 & 53.3 & 5 & 71.4 & \\
\hline
\end{tabular}

Table 4. Impact of Acetylator phenotype on clinical outcomes in 22 TB patients

\begin{tabular}{|c|c|c|c|c|c|c|}
\hline \multirow[t]{2}{*}{ Variable } & \multirow[t]{2}{*}{ Outcome* } & \multicolumn{2}{|c|}{ Slow Acetylator } & \multicolumn{2}{|c|}{ Fast Acetylator } & \multirow[b]{2}{*}{$p$-value } \\
\hline & & $\mathbf{n}$ & $\%$ & $\mathbf{n}$ & $\%$ & \\
\hline \multirow[t]{3}{*}{ Therapeutic outcome } & Improved & 11 & 73.3 & 6 & 85.7 & 0.724 \\
\hline & Stable & 3 & 20.0 & 1 & 14.3 & \\
\hline & Death & 1 & 6.7 & 0 & 0.0 & \\
\hline \multirow[t]{2}{*}{ Sputum culture control } & Negative & 13 & 86.7 & 6 & 85.7 & 0.952 \\
\hline & Positive & 2 & 13.3 & 1 & 14.3 & \\
\hline \multirow[t]{2}{*}{ Renal failure } & No side effect & 14 & 93.3 & 7 & 100.0 & 0.484 \\
\hline & Yes & 1 & 6.7 & 0 & 0.0 & \\
\hline \multirow[t]{2}{*}{ Peripheral neuropathy } & No side effect & 7 & 46.7 & 7 & $' 100.0$ & 0.015 \\
\hline & Yes & 8 & 53.3 & 0 & 0.0 & \\
\hline \multirow[t]{2}{*}{ Anaemia } & No side effect & 14 & 93.3 & 6 & 85.7 & 0.563 \\
\hline & Yes & 1 & 6.7 & 1 & 14.3 & \\
\hline
\end{tabular}

\section{Discussion}

In our study, by taking the cut-off point at $70 \%$ AcINH according to the instruction of the method used (Ellard \& Gammon, 1973, pp.201-210), the bimodality gave $75 \% \mathrm{SA}$ and $25 \% \mathrm{FA}$ in the healthy group or $68.2 \% \mathrm{SA}$ and $31.8 \% \mathrm{FA}$ in TB group. However, a scrutiny of the distribution (Fig.1) showed two cut-off limit points at $45 \%$ and $75 \%$, which split the sample in three clusters corresponding to a trimodal distribution with $30 \% \mathrm{SA}$, $45 \%$ IA, and $25 \%$ FA for the healthy group and $13.6 \%$ SA, $54.5 \%$ IA and $31.8 \%$ FA for the TB group.

In many studies, the acetylation phenotype of isoniazid has been found to be bimodal although some discrep- ancies had been raised over the proportions of slow and fast acetylators. In some people, the SA group was dominant while in others the dominant was FA cluster. For example, Bouayad and al.(1982, pp.401-407) in Morocco found $41 \% \mathrm{SA}$ as compared to $59 \% \mathrm{FA}$, while the study of Moussa et al.(2002, pp.548-555) using the HPLC method found a bimodal distribution with $61.8 \%$ SA and $38.2 \% \mathrm{FA}$, the inverse of Bouayad's data in the same population. In black South Africans, Bach and al. (1976,pp.1132-1134) also obtained 41\% SA against 59\% FA. The study of Parkin et al.(1997, pp.1717-1722), however, described a trimodal distribution in South African TB patients where the NAT2*12A allele which codes for fast acetylation had a high frequency. 
Looking through different studies, it appears clearly that the accurate cut-off limit for differentiating slow, intermediate, and fast acetylator depends on the method used. The intermediate group affects significantly the bimodal profile depending where the skew is forwarded to. For example in our study, if the $45 \%$ cut-off is the only point considered, the distribution could be considered bimodal leading to $25 \%$ of SA and $75 \%$ of $\mathrm{FA}$ for the entire pooled sample; the proportions would reverse if the cutoff limit is $70 \%$ AcINH.

In the literature, differing results have been obtained concerning the influence of age, sex, and TB or HIV illness on the pharmacokinetics of isoniazid and on the acetylator profile. Some data demonstrated HIV/AIDS status or gender had no significant effect on the concentrations of isoniazid in plasma (Comte et al., 2002, pp.2358-2364; Kimerling et al.,1998, pp.1178-1183), while other studies showed a significant reduction of isoniazid and rifampin concentrations in serum of non-HIV-infected patients with tuberculosis who received directly observed therapy (Peloquin et al., 1997, pp.2670-2679). In our study, even though no significant correlation was found for the influence of TB or HIV illness on the acetylator phenotype, a rough analysis of the trends indicated that the ratios of $\%$ AcINH tend to shift towards higher values in TB patients as compared to a healthy population without deteriorating the trimodal profile, meaning that the effect of TB or HIV would modify only the proportions between slow, intermediate and fast categories. In addition, the sex and age did not affect significantly the acetylator phenotype profile, but since the acetylation is a genetically controlled metabolism, their influence cannot be absolutely ruled out as the sample size may limit definitive interpretation.

After initiating a short treatment course of two months with RHZE, about $80 \%$ of patients had their sputum smear culture negative. The three patients who remained in stable condition without significant improvement with smear culture positive may lead to think of multiple resistant drug (MRD)-TB. This correlates with the figures reported countrywide $(\mathrm{MoH}, 2012)$ that showed a high treatment success rate for the new smear-positive TB patients $(88.4 \%)$ and for MDR-TB cases $(88 \%)$. There were no significant differences between slow and fast acetylators. On the other hand, the occurrence of adverse drug reactions was in agreement with other studies which demonstrated that the peripheral neuropathies are more likely to occur early in patients having a low speed of acetylation, suggesting that INH blood levels are high in this group (Comte et al., 2002, pp.2358-2364; Yee et al.,2003, pp.1472-1477). It is obvious that patients with a fast speed of acetylation may experience hepatitis com- plications long term as reported in the literature (Mitchell et al.,1975, pp.70-79; Steele et al.,1991, pp.465-71; Huang et al., 2002, pp.883-889), but it was not possible to observe it in our short course treatment time of two months.

\section{Limitations}

The present study has been undertaken to figure out the polymorphism type of isoniazid acetylation in Rwandese. A small sample size of 42 participants was used, and this may limit the generalization of the findings at the national level. For the control group, students of former National University of Rwanda were selected to avoid the impact of unknown health conditions. Nevertheless the results obtained are comparable to those obtained in large populations. For example, the rate of coinfection with TB and HIV (27.27\%) found in this small sized population was close to the $28 \%$ value reported for the larger population of Rwanda (MoH, 2012).

\section{Conclusion}

Notwithstanding the limitation of the small size of the sample and the accuracy of the spectrophotometric method used, this study shows that Isoniazid Acetylator Profile in Rwandese can be described better by a trimodal distribution. The phenotype pattern may be influenced somehow by TB infection or HIV infection but the clinical implication remains under the genetic control only. The slow metabolisers should be monitored for peripheral neuropathies and skin allergies.

\section{Acknowledgements}

We are grateful to all participants for their complete cooperation and we appreciated the technical assistance from the nursing body of the CHUB/Internal Medicine Service as well as the skilled help of the technicians of Medical Laboratory. Tablets of INH have been provided by the former National Program for Struggle against Leprosy and Tuberculosis (PNILT).

\section{Conflict of interest and source of funding}

There is no any conflict of interest; the study has been conducted as graduate dissertation under the University fund.

\section{References}

Bach, P.H., Higgens-Opitz, S.B., Bima, B.C., \& Leary, W.P. (1976). Isoniazid acetylator status of black South African tuberculosis patients. S Afr Med J, 50(29),1132-1134.

Bouayad, Z., Chevalier, B., Maurin, R., \& Bartal, M. (1982). 
Acetylation phenotype of isoniazid in Morocco. Preliminary study of 100 cases. Rev. Fr. Mal. Resp, 10(6), 401-407.

Comte, J.E., Jeffrey, Jr., Goblen, A., Kipps, J., Duncan, S., McKenna, E., \& Zurlinden, E. (2002). Effect of gender, AIDS and acetylator status on intrapulmonary concentration of isoniazid. Antimicrob Agents Chemother, 46(8), 2358-2364.

Eidus, L. \& Hodgkin, M.M. (1973). Screening of Isoniazid Inactivators. Antimicrob. Agents Chemother, 3(1),130-133. doi:10.1128/AAC.3.1.130.

Ellard, G.A. \& Gammon, P.T. (1973). Determination of the acetylator phenotype from the ratio of the urinary excretion of acetylisoniazid to acid-labile isoniazid: a study in Finnish Lapland. Tubercle, 54(3), 201-210.

Ellard, G.A. (1976).Variations between individuals and populations in the acetylation of isoniazid and its significance for the treatment of pulmonary tuberculosis. Clin Pharmacol Ther, 19, 610-625.

GHO (Global Health Observatory). (2013). Tuberculosis (TB) Retrieved from http://www.who.int/gho/tb/en

GPS-TB(The Global Plan to Stop TB) (2013). Retrieved from http://www.stoptb.org/global/plan .

Huang, Y.S., Chern, H.D., Su, W.J., Wu, J.C., Lai, S.L., Yang, S.Y., Chang, F.Y., \& Lee, S.D. (2002). Polymorphism of the $\mathrm{N}$-acetyltransferase 2 gene as a susceptibility risk factor for antituberculosis drug induced hepatitis. Hepatology, 35:883889.

Junichi,A., Masako,O., Ryuji, K., Soichiro, Y., Takayuki, N., Kazunari,T., Yasuhisa, O., Tetsuya, T., Sayaka, K., Yasushi, F., \& Ichiro, K..(2013). NAT2 genotype guided regimen reduces isoniazid-induced liver injury and early treatment failure in the 6-month four-drug standard treatment of tuberculosis: A randomized controlled trial for pharmacogenetics-based therapy. Eur J Clin Pharmacol, 69,1091-1101. doi :10.1007/ s00228-012-1429-9.

Kaishusha, M.P., Kadima,N.J.(2009) Treatment adhesion and factors affecting it at the Kadutu Clinic (Democratic Republic of the Congo). Cahiers Sante,19,205-15.(in French).

Kimerling, M.E., Phillips, P., Patterson, P. (1998) Hall M, Robinson CA, \& Dunlap NE. Low serum antimycobacterial drug levels in non-HIV-infected tuberculosis patients. Chest, 113,1178-1183.

Kinzig-Schippers, M., Tomalik-Scharte, D., Jetter, A., Scheidel, B., Jakob, V., Rodamer, M., Cascorbi, I., Doroshyenko, O., Sörgel, F., \& Fuhr, U. (2005). Should We Use N-Acetyltransferase Type 2 Genotyping To Personalize Isoniazid Doses? Antimicrob. Agents Chemother, 49(5),1733-1738. doi: 10.1128/ AAC.49.5.1733-1738.2005.

Mitchell, J.R., Thorgeirssonup, J., Black, M., Timbrell, J.A., Snodgrass, W.R., Potter, W.Z., Jollow, H.R., \& Keiser, H.R. (1975). Increased incidence of isoniazid hepatitis in rapid acetylators: possible relation to hydrazine metabolites. Clin Pharmacol Ther, 18 (1):70-79.
$\mathrm{MoH}$, (2012). The Human resources for health program in Rwanda. Retrieved from www.hrhconsortium.moh.gov.rw/abouthrh/program-overview/

Moussa,A.L., Khassouani,C.E., Hüe B.,Jana,M.,Begaud,B.,Soulaymani,R..(2002). Determination of the acetylation phenotype in Moroccan tuberculosis patients using isoniazid as metabolic probe. Int.J Clin Pharm Therap, 40(12):548-555.

$\mathrm{NIH}, 2013$. Guidelines for the Use of Antiretroviral Agents in HIV-1-Infected Adults and Adolescents. Retrieved from https:/ / aidsinfo.nih.gov/hiv-aids-health-topics

Parkin, D.P., Vandenplas, S., Both, A.F.J., Seifart, H.I., van Helden, P.D., van der Walt, B.J., Donald, P.R., \& van Jaarsveld, P.P. (1997). Trimodal of isoniazid elimination: Phenotype and genotype in patients with tuberculosis. Am J Resp Crit, 155(5),1717-1722.

Pasipanodya, J.G., Srivastava, S., \& Gumbo, T.(2012). Meta-analysis of clinical studies supports the pharmacokinetic variability hypothesis for acquired drug resistance and failure of antituberculosis therapy. Clin Infect Dis, 55(2),169-177.

Patin, E., Harmant, C., Kidd, K.K., Kidd, J., Froment, A., Mehdi, S.Q., Sica, L., Heyer, E., \& Quintana-Murci, L. (2006). Sub-Saharan African coding sequence variation and haplotype diversity at the NAT2 gene. Hum Mutat, 27(7), 720.

Peloquin, C.A., Jaresko, G.S., Yong, C.L., Keung, A.C., Bulpitt, A.E., \& Jelliffe, R.W. (1997).

Population pharmacokinetic modeling of isoniazid, rifampin, and pyrazinamide. Antimicrob Agents Chemother, 41,26702679.

Steele, M.A., Burk, R.F., \& DeaPrez, R.M.(1991). Toxic hepatitis with isoniazid and rifampin. A meta-analysis. Chest, 99,465-71.

Van de Perre, P., Simonon, A., Msellati, P., Hitimana,D., Vaira, D., Bazubagira, A., Van Goethem, C., Stevens, A., Karita, E., Sondag-Thull, D., Dabis, F., \& Lepage, P.(1991). Postnatal Transmission of Human Immunodeficiency Virus Type 1 from Mother to Infant .A Prospective Cohort Study in Kigali, Rwanda. N Engl J Med, 325, 593-598. doi:10.1056/ NEJM199108293250901.

Weber, W. W. \& Brenner, W. A (1974). Filter Paper Method for Determining Isoniazid Acetylator Phenotype. Am J Hum Genet, 26, 467-473.

WHO,(2010). Guidelines for treatment of tuberculosis, 4th ed. Retrieved from http://www.who.int/tb/publications/

WHO,(2013a). Tuberculosis and HIV. Retrieved from http:// www.who.int/hiv/topics/tb/en

WHO,(2013b). Multidrug resistant tuberculosis. Retrieved from http://www.who.int/tb/challenges/mdr/en

Yee, D., Valiquette, C., Pelletier, M., Parisien, I., Rocher, I., \& Menzies, D. (2003). Incidence of serious side effects from first-line antituberculosis drugs among patients treated for active tuberculosis. Am J Respir Crit Care Med, 167, 1472 1477. 